\title{
Evaluación del impacto de la pandemia COVID-19 en el diagnóstico y tratamiento de pacientes con cáncer de mama tratadas en Clínica Alemana de Santiago
}

\author{
Carolina Barriga S. ${ }^{1}$, Jamile Camacho N. ${ }^{1}$, Eugenio Román L. ${ }^{1}$, Tatiana Retamal R. ${ }^{1}$, \\ Fernando Cádiz V.' , Marcela Gallego A. ' y Daniel Carvajal H.
}

Evaluation of the impact of the COVID-19 pandemic in the diagnosis and treatment of breast cancer patients treated at Clínica Alemana de Santiago

Introduction: Due to SARS-CoV-2 pandemic there has been a delay in oncological diagnosis and treatments potentially increasing mortality. Aim: To evaluate the effects of the pandemic in patients treated in Clinica Alemana with recent diagnosis of breast cancer, comparing the reason of consultation, stage and treatment to a similar time frame in 2019. Materials and Method: This is a retrospective, descriptive and quantitative study. Analyzing patients registry from April 1st to July 31st, 2020 compared with same time frame of 2019. Retrospective analysis of our database searching for reason of consultation, stage and treatment. Data analysis using STATA, T student test and Fisher exact test, considering significant a $p<0.05$. Results: N156, $70(44.87 \%)$ consultations in the 2020 -time frame versus $86(55.13 \%)$ in 2019 $(p=0.1)$. Mean age 55 years versus $58(p=0.38)$. Palpable tumor 25 versus $29(p=0.86)$. There was a difference in patients consulting due to personal breast oncological background, 0 versus $6(p=0.033)$ or familiar breast oncological background 0 versus $6(p=0.033)$. Breast images control 39 versus 53 $(p=0.5)$. In Pandemic more Her 2 tumors were diagnosed 11 versus $2(p=0.006)$. No differences in stages were observed. Upfront surgical treatment in 49 versus 71 patients $(p=0.85)$ and neoadjuvant hormonal treatment 7 versus $1(\mathrm{p}=0.058)$. Conclusions: In the pandemic time frame there were less consultations due to breast cancer. The majority of the patients came because of a palpable tumor or breast image control without a significant difference compared with a similar time frame in previous year. There were less surgeries and more neoadjuvant hormonal treatments.

Key words: SARS-CoV-2; COVID-19; breast cancer; delays in cancer diagnosis; changes in treatment plans.

\section{Resumen}

Introducción: Debido a la pandemia COVID-19 se ha visto un retraso en diagnósticos de enfermedades oncológicas, cambio de tratamientos y aumento en mortalidad. Objetivo: Evaluar efectos de la pandemia en pacientes de Clínica Alemana de Santiago con diagnóstico reciente de cáncer de mama, comparadas con igual periodo año 2019. Materiales y Método: Estudio cuantitativo, retrospectivo, tipo descriptivo. Período 1 de abril - 31 de julio de 2020 comparado con igual periodo de año 2019. Revisión de nuestra base de datos, comparando motivo de consulta, estadio y tratamiento. Análisis estadístico con programa STATA, test T student y test exacto de Fisher. Consideramos significativo $\mathrm{p}<0,05$. Resultados: Total 156 pacientes, $70(44,87 \%)$ consultaron en periodo señalado año 2020 versus $86(55,13 \%)$ en $2019(\mathrm{p}=0,1)$. Edad promedio 55 años versus $58(\mathrm{p}=0,38)$. Consulta por nódulo palpable de mama, 25 versus $29(\mathrm{p}=$ $0,86)$. Hubo diferencias en pacientes que consultaron por antecedentes familiares de cáncer de mama, 0 versus $6(\mathrm{p}=0,033)$, y en pacientes con antecedente personal de cáncer de mama, 0 versus $6(\mathrm{p}=0,033)$. Consulta por control imagenológico fue 39 versus $53(\mathrm{p}=0,5)$. En época de pandemia hubo más tumores Her 2, 11 versus $2(\mathrm{p}=0,006)$. Por estadios, no hubo diferencia. Por tratamiento indicado, cirugía fue de 49 pacientes versus $71(\mathrm{p}=0,85)$. Hormonoterapia neoadyuvante 7 versus $1(\mathrm{p}=0,058)$. Conclusiones: En periodo de pandemia hubo menor consulta por cáncer de mama. La mayoría por control imagenológico y lesiones palpables, sin diferencia en estos grupos entre ambos periodos. Hubo menos cirugías y más tratamientos con hormonoterapia neoadyuvante.

Palabras clave: pandemia; SARS-CoV-2; COVID-19; cáncer de mama; demora en tratamiento. 


\section{Introducción}

La enfermedad del coronavirus COVID-19, fue descrita como un virus parecido a SARS-CoV, en la provincia de Hubei en China en diciembre de 2019¹. El 11 de marzo de 2020 la OMS la declaró como pandemia. Actualmente se conoce como SARS-CoV-2 ${ }^{2}$.

Un fenómeno visto a nivel mundial, es que las personas no están consultando en centros de salud, por temor a contraer el SARS-CoV-2, pudiendo esto aumentar la tasa de morbilidad y mortalidad para otras patologías, lo que se ha llamado la "segunda pandemia". El objetivo de nuestro estudio es evaluar la situación en nuestra clínica, comparando pacientes con diagnóstico reciente de cáncer de mama en un periodo determinado de la pandemia con igual periodo del año anterior y evaluar si hay algún retraso en el diagnóstico y/o algún cambio en el tratamiento. No analizamos la mortalidad, ya que para eso se necesita más seguimiento. La importancia de este estudio es que nos permite tener un diagnóstico local de nuestro centro y poder así planificar estrategias de manejo de nuestras pacientes pospandemia.

\section{Materiales y Método}

Se realizó un estudio cuantitativo, retrospectivo, de tipo descriptivo de pacientes con diagnóstico reciente de cáncer de mama, tratadas en el Centro de la Mama de Clínica Alemana de Santiago, comparando el periodo de pandemia del 1 de abril de 2020 al 31 de julio de 2020 con igual periodo del año anterior. Los datos fueron obtenidos de la base de datos de nuestro centro. Se realizó análisis estadístico con el programa STATA, usando test $\mathrm{t}$ de Student y test exacto de Fisher. Se consideró significativo $p<0,05$. Se compara edad, motivo de consulta (lesión palpable, lesión en la piel o pezón, antecedente personal o familiar de cáncer de mama, hallazgo imagenológico), diagnóstico histológico y molecular, estadio y tratamiento. El estudio y análisis de los datos fue aprobado por el comité de ética de nuestra institución.

\section{Resultados}

El total de pacientes tratadas en estos periodos fue $156,70(44,87 \%)$ consultaron en el periodo señalado de la pandemia del año 2020 y $86(55,13 \%)$ en $2019(p=0,1)$. A pesar de que el $p$ no es significativo, sí es sugerente de significación el haber tratado menos pacientes durante el período de la pandemia. La edad promedio de las pacientes fue 55 años el año 2020 y 58 años el $2019(\mathrm{p}=0,38)$.

En relación al motivo de consulta no hubo diferencia si fue por nódulo palpable en la mama, 25 pacientes en época pandemia y 29 no pandemia $(p=0,86)$, tampoco por lesión palpable en la axila, 2 en cada periodo $(p=1)$, tampoco por cambio de piel, 2 en 2020 y 0 en $2019(\mathrm{p}=0,2)$, secreción por el pezón, 0 en 2020 y 2 en 2019 ( $p=0,5)$, ni dolor, 0 en 2020 y 1 en $2019(p=1)$. Sí hubo diferencias en pacientes que consultaron por antecedentes familiares de cáncer de mama, época pandemia 0 y 6 en no pandemia $(p=0,033)$, como también en pacientes con antecedente personal de cáncer de mama, 6 año 2019 y 0 año $2020(p=0,033)$. La consulta por control de imágenes fue 39 en época pandemia y 53 en no pandemia $(p=0,5)($ Tabla 1$)$.

Tabla 1. Motivo de consulta referidos por pacientes

\begin{tabular}{|lccc|}
\hline & Año 2020 & Año 2019 & Valor p \\
Nódulo mamario palpable & 25 & 29 & 0,86 \\
Nódulo axilar palpable & 2 & 2 & 1,0 \\
Cambios en la piel & 2 & 0 & 0,20 \\
Secreción por el pezón & 0 & 2 & 0,50 \\
Dolor & 0 & 1 & 1,0 \\
Antecedente familiar cáncer de mama & 0 & 6 & 0,03 \\
Antecedente personal cáncer de mama & 0 & 6 & 0,51 \\
Hallazgo imagenológico & 39 & 53 & 0,70 \\
Otros & 4 & 3 & 102 \\
Total & 72 & & \\
\hline
\end{tabular}


ARTíCULO ORIGINAL

No hubo diferencia en el diagnóstico histológico entre periodo pandemia año 2020 e igual periodo año 2019 (cáncer ductal infiltrante 53 y 61, cáncer lobulillar infiltrante 6 y 5, cáncer ductal in situ 9 y 14 , otros 2 y 6 respectivamente) (Tabla 2). Evaluados los cánceres infiltrantes por el diagnóstico molecular, se vio que en época pandemia hubo más diagnóstico de tumores Her 2, 11 y 2 en no pandemia $(p=0,006)$, no hubo diferencia en el resto de los diagnósticos moleculares comparando el periodo del año 2020 con 2019 (Tabla 3). En la evaluación por estadios al momento del diagnóstico, no hubo diferencia entre el periodo 2020 y 2019, siendo la mayoría de las pacientes con cánceres en estadios precoces (Tabla 4).

En relación con el tratamiento indicado, vimos que la cirugía el año 2020 fue realizada en 49 pacientes y año 2019 de $71(\mathrm{p}=0,85)$, a pesar de no ser significativo, es sugerente de significación el haber operado menos pacientes durante la pandemia. No hubo diferencia en las indicaciones de quimioterapias neoadyuvantes (11 en periodo de pandemia y 12 igual periodo año 2019). La hormonoterapia neoadyuvante se indicó en época de pandemia en 7 pacientes y no pandemia $1(\mathrm{p}=0,058)$, siendo sugerente de significación el haber tenido mayor número de pacientes con hormonoterapia neoadyuvante durante el periodo de pandemia. No hubo diferencias en relación con terapias paliativas ( 3 en periodo año 2020 y 2 año 2019) (Tabla 5).

\section{Discusión}

El coronavirus corresponde a una gran familia de virus, que pueden causar desde leves resfríos hasta cuadros respiratorios severos. Algunos coronavirus son zoonóticos, es decir, se pueden transmitir de animales a humanos. La tasa de mortalidad publicada en China es de $2,3 \% \%^{3}$, otro estudio del Reino Unido publica una tasa de $26 \%$ en pacientes hospitalizados ${ }^{4}$. Estas cifras varían según el grupo etario, comorbilidades y nivel de desarrollo del sistema de salud de cada país. En la actualidad esta enfermedad no tiene ningún tratamiento específico, hay varias vacunas que ya se están administrando.

Los datos muestran que la pandemia COVID-19 ha provocado un retraso en el diagnóstico de pacientes con cáncer, ya que se ha visto un menor número de casos diagnosticados en comparación con igual período del año $2019^{5}$. Nuestra serie incluyó pacientes con diagnóstico reciente de cáncer de mama entre 1 abril de 2020 y 31 de julio de 2020, periodo que fue el más representativo de la pandemia en
Tabla 2. Diagnóstico histológico

\begin{tabular}{|lccc|}
\hline & Año 2020 & Año 2019 & Valor p \\
Cáncer ductal infiltrante & 53 & 61 & 0,46 \\
Cáncer lobulillar infiltrante & 6 & 5 & 0,54 \\
Cáncer ductal in situ & 9 & 14 & 0,65 \\
Otro & 2 & 6 & 0,13 \\
Total & 70 & 86 & \\
\hline
\end{tabular}

Tabla 3. Diagnóstico molecular en cánceres infiltrantes

\begin{tabular}{|lccc|}
\hline & Año 2020 & Año 2019 & Valor p \\
Luminal A & 31 & 43 & 0,29 \\
Luminal B & 13 & 21 & 0,32 \\
Her 2 & 11 & 2 & 0,06 \\
Triple negativo & 6 & 4 & 0,51 \\
Total & 61 & 70 & \\
\hline
\end{tabular}

Tabla 4. Estadios al momento del diagnóstico

\begin{tabular}{|lccc|}
\hline & Año 2020 & Año 2019 & Valor p \\
\hline Estadio 0 & 9 & 16 & \\
Estadio I & 33 & 41 & \\
Estadio II & 20 & 18 & \\
Estadio III & 2 & 7 & \\
Estadio IV & 6 & 4 & \\
Total & 70 & 86 & 0,35 \\
\hline
\end{tabular}

Tabla 5. Tratamiento inicial

\begin{tabular}{|lccc|}
\hline & Año 2020 & Año 2019 & Valor p \\
Cirugía & 49 & 71 & 0,08 \\
Quimioterapia neoadyuvante & 11 & 12 & 0,82 \\
Hormonoterapia neoadyuvante & 7 & 1 & 0,058 \\
Paliativo & 3 & 2 & 0,65 \\
Total & 70 & 86 & \\
\hline
\end{tabular}

Santiago. En comparación con el año anterior, tuvimos menor número de pacientes diagnosticadas, lo cual explicamos por el temor de ellas a acudir a un centro de salud y contagiarse con SARS-CoV-2, ya que en nuestro centro no se suspendieron las atenciones de consulta, diagnóstico ni tratamiento. Estos resultados se mantienen al ser comparados con el 
mismo período del año 2018, en que tratamos 80 pacientes con diagnóstico reciente de cáncer de mama.

Otro efecto del COVID-19 es que ha modificado los planes de tratamiento en algunos pacientes oncológicos. Algunos paneles de expertos han publicado recomendaciones en relación a este tema, como el consenso de expertos de European Society of Medical Oncology (ESMO), sobre por ejemplo en qué pacientes diferir las cirugías o tratamientos de quimioterapia ${ }^{6}$. Un estudio francés publicó, que, en pacientes oncológicos con terapia sistémica, en un $27 \%$ de los casos hubo modificación en el tratamiento, llegando a $41 \%$ en los cánceres de pulmón y cabeza y cuello ${ }^{7}$. Será importante evaluar a futuro si estos cambios de terapias tendrán un impacto en la sobrevida de los pacientes con cáncer. En nuestra serie hubo un menor número de cirugías y mayor número de indicaciones de hormonoterapia neoadyuvantes, en relación con el periodo anterior (ambos estadísticamente sugerente de significación), indicaciones que fueron evaluadas caso a caso en nuestro comité multidiciplinario de mama. La mayoría eran pacientes mayores de 75 años con tumores luminal A, en que se prefirió iniciar el tratamiento con terapia hormonal, hasta que mejoraran las condiciones de la pandemia para una cirugía.

También se ha visto un aumento de la mortalidad en pacientes oncológicos que han contraído este virus. Se sabe que el cáncer es una condición médica que aumenta el riesgo de tener una enfermedad severa de COVID-19 y que al contraerlo tienen peor pronóstico ${ }^{8}$. En pacientes con cáncer, la mortalidad por COVID-19 descrita es entre 11 y 33\%, dependiendo de la edad, sexo (masculino tiene peor pronóstico) y comorbilidades 9 .

El estado de inmunosupresión en que se encuentran algunos pacientes con cáncer (causado por la misma enfermedad o por el tratamiento), incrementa su riesgo de infección en comparación a la población general. Pacientes que han recibido quimioterapia o han sido sometidos a cirugía 30 días antes de contagiarse con COVID-19, tienen mayor riesgo de tener complicaciones severas. Esto puede llevar además como consecuencia un retraso en sus tratamientos, lo que puede afectar sus pronósticos. Sin embargo, se ha visto un aumento en la mortalidad no sólo en pacientes oncológicos activos, sino también en pacientes con antecedentes de haber tenido un cáncer. La mortalidad reportada de un paciente COVID-19 en ventilación mecánica invasiva (VMI) no oncológico es $29,5 \%$, con antecedente de cáncer $44,3 \%$ y con cáncer activo un $42,3 \%$.

Un fenómeno que se vio a nivel mundial, incluso en Chile, es que disminuyó el número de pacientes que consultaban en los centros de salud, retrasando sus controles y no consultando por síntomas nuevos, por temor a contraer el SARS-CoV-2. Esto puede provocar otra crisis de salud, al hacer diagnósticos y tratamientos tardíos, pudiendo aumentar la tasa de morbilidad y mortalidad para estas patologías. En nuestra serie no hubo diferencias en los estadios al momento del diagnóstico al compararlo con el año 2019, por lo que no tuvimos mayor número de cánceres avanzados. Esto será interesante analizarlo con los datos del año 2021.

El cáncer es una enfermedad cuyo pronóstico depende en gran parte del oportuno diagnóstico y tratamiento. El miedo de los pacientes a contagiarse en los centros de salud, ha llevado a que no hagan los tamizajes, retrasando el diagnóstico y oportuno tratamiento de la enfermedad. En algunos cánceres, un retraso de tres meses en el diagnóstico puede tener un impacto en la sobrevida. Algunos estudios han demostrado que el tiempo en que un tumor mamario dobla su tamaño, varía entre 45 a 260 días. Es un rango amplio que no nos ayuda a determinar el real efecto del retraso del diagnóstico en la sobre$v i^{11}{ }^{11}$. También hay estimaciones que, en un plazo de 6 meses, hasta un 50\% de los tumores mamarios podrían aumentar en más de un $\mathrm{cm}$ su tamaño ${ }^{11,12}$.

A esto se puede agregar que muchos centros de salud en el mundo difirieron los tamizajes, las cirugías y terapias adyuvantes para dar prioridad a la atención de pacientes COVID-19 hospitalizados. Este no fue el caso de nuestra institución en que las cirugías oncológicas tuvieron prioridad.

Un modelo creado por el Instituto Nacional del Cáncer (NCI) de USA, predijo que, en las próximas décadas en ese país, se producirán más de 10.000 muertes extras por cáncer de colon y mama, como resultado de la disminución de tamizajes, retraso del diagnóstico y tratamientos. Esto principalmente en los próximos dos años y corresponde a un aumento del $1 \%$ en las muertes esperadas ${ }^{13}$. Así mismo, el NCI estima que desde marzo ha habido una disminución de $75 \%$ de mamografías realizadas en USA.

Un estudio realizado en Inglaterra, estima que la mortalidad por cáncer de mama aumentará entre $8 \%$ y $9,6 \%$ en un periodo de hasta 5 años ${ }^{14}$.

En países como Escocia, Italia y España, se han publicado aumento de tasas de mortalidad por patologías no COVID. En algunas regiones de España las tasas de mortalidad casi han duplicado, con sólo una fracción del aumento atribuible oficialmente a COVID.

El diagnóstico de cáncer de mama se realiza básicamente por 3 maneras: tamizaje, controles clínicos periódicos y consulta de pacientes por lesiones ma- 
marias palpables. En Chile según datos de Globocan el año 2018 se diagnosticaron 5.393 nuevos casos de cáncer de mama ${ }^{15}$.

\section{¿Cuál es la situación actual en Chile?}

Según la Asociación de Clínicas de Chile, la atención de urgencias no relacionadas a COVID-19, bajó un 51\% a partir de mediados de marzo. Además, disminuyeron también las consultas por otras enfermedades no asociadas al virus. Esto podría tener como consecuencia que los cánceres se diagnostiquen en estadios más avanzados con un aumento probable de quimioterapias neoadyuvantes, además de un aumento en la mortalidad, costos y sobrecarga posterior del sistema de salud ${ }^{17}$.

En Clínica Alemana de Santiago (CAS) las consultas médicas y actividad quirúrgica cayeron un $70 \%$ en ese período. La tasa de diagnóstico de cáncer en la población que se atiende en la clínica bajó un 52\%, respecto a igual periodo del año 2019, el de próstata un $70 \%$ y el de mama un $40 \%$.

En nuestro estudio vimos una baja no significativa en los nuevos cánceres de mama diagnosticados en el periodo de pandemia ( 1 de abril al 31 de julio de 2020) al compararlos con el mismo periodo del año 2019. Al contrario de lo que podríamos haber esperado, no tuvimos disminución en los tumores no palpables (diagnosticados por hallazgos imagenológicos) ni aumento de los palpables, lo que indica que nuestro grupo de pacientes se siguió controlando, lo cual se podría explicar porque en nuestro medio las pacientes tienen mayor acceso a conseguir horas de consulta y exámenes.

En el diagnóstico histológico no hubo diferencias en ambos periodos, sí tuvimos más tumores Her 2 en período de pandemia, la mayoría de estos no palpables.

\section{Conclusión}

Durante el periodo de pandemia SARS-CoV-2 vimos una menor consulta de pacientes por cáncer de mama. La mayoría de las pacientes consultó por control imagenológico y en segundo lugar por lesiones palpables en la mama, no habiendo diferencia en estos grupos entre ambos periodos. Con relación al tratamiento, tuvimos menor número de cirugías en época de pandemia y mayor número de pacientes con homonoterapia neoadyuvante.

\section{Responsabilidades éticas}

Protección de personas y animales. Los autores declaran que para esta investigación no se han realizado experimentos en seres humanos ni en animales.

Confidencialidad de los datos. Los autores declaran que en este artículo no aparecen datos de pacientes.

Conflictos de interés: no hay.

\section{Bibliografía}

1. Osama M Al-Quteimat, Mustafa A, et al. The Impact of the COVID-19 Pandemic on Cancer Patients. Am J Clin Oncol. 2020;43:452-5. doi: 10.1097/ COC.0000000000000712.

2. Casella D, Fusario D, Cassetti D, Miccoli S, Pesce AL, Bernini A, et al. The patient's pathway for breast cancer in the COVID-19 era: An Italian single-center experience. The Breast Journal. 2020;26:1589-92. doi: 10.1111/ tbj. 13958.

3. Zunyou $\mathrm{Wu}, \mathrm{McGoogan} J$. Characteristics of and Important Lessons From the Coronavirus Disease 2019 (COVID-19) Outbreak in China. Summary of a Report of 72,314 Cases From the Chinese Center for Disease Control and Prevention. JAMA. 2020;323:1239-42. doi: 10.1001/ jama.2020.2648.

4. Docherti A, Green C, Hardwick H, Plus $\mathrm{R}$, Norman L, Holden K, et al. Features of 20133 UK patients in hospital with covid-19 using the ISARIC WHO Clinical Characterisation Protocol: prospective observational cohort study. BMJ 2020;369:1-12. doi: https://doi. org/10.1136/bmj.m1985.

5. Dinmohamed AG, Visser O, Verhoeven R, Louwman M, van Nederveen F, Willems $\mathrm{S}$, et al. Fewer cancer diagnoses during the COVID-19 epidemic in the Netherlands. The Lancet Oncol. 2020;21:750-1. doi: https://doi.org/10.1016/S14702045(20)30265-5.

6. Curiguliano G, Banerjee S, Cervantes A, Garassino MC, Garrido P, Girard J, et al. Managing cancer patients during the COVID-19 pandemic: an ESMO multidisciplinary expert consensus. Ann
Oncol. 2020;31:1320-35. doi: https://doi. org/10.1016/j.annonc.2020.07.010.

7. Joly F. LBA2637: Impact of the COVID-19 pandemic on management of medical cancer treatments and psychological consequence for the patients: the COVIPACT study. Reportet at the European Society of Medical Oncology (ESMO) virtual congress 2020. Annals of Oncology 2020;31:1142-215.

8. Liang W, Guan W, Chen R, Wang W, Li $\mathrm{J}, \mathrm{Xu} \mathrm{K}$, et al. Cancer patients in SARS$\mathrm{CoV}-2$ infection: a nationwide analysis in China. The Lancet Oncol. 2020;21:335-7.

9. Garassino MC, Whisenant J, Li-Ching Huang, Trama A, Torri V, Agustoni F, et al. COVID-19 in patients with thoracic malignancies (TERAVOLT): first results of an international, registry-based, cohort study. The Lancet Oncol. 2020;21:914-22.

10. Palmieri C, Turtle L, Docherty A, Harrison 


\section{ARTÍ́CULO ORIGINAL}

E, Drake T, Greenhalf B, et al. \#3470 Prospective data of first 7,026 hospitalized patients with Cancer and COVID-19 derived from the COVID-19 Clinical Information Network and International Sever Acute Respiratory and Emerging Infections Consortium WHO Coronavirus Clinical Characterisarion Consortium. Reported at the European Society of Medical Oncology (ESMO) virtual congress 2020.

11. Bleicher RJ. Timing and delays in breast cancer evaluation and treatment. Ann Surg
Oncol. 2018;25:2829-38. doi: 10.1245/ s10434-018- 6615-2.

12. Osservatorio nazionale screening Rapporto 2018. Available online at: https://www. osservatorionazionalescreening.it/ content/ rapporto (accessed April 3, 2020).

13. Sharpless N. Editorial Covid -19 and cancer. Science 2020;368:1290-31. doi: 10.1126

14. Maringe C, Spicer J, Morris M, Purushotham A, Nolte E, Sullivan R, et al. The impact of the COVID-19 pandemic on cancer deaths due to delays in diagnosis in England, UK: an national, populationbase modelling study. Lancet Oncol. 2020;21:1023-34. Published Online July 20, 2020 https://doi.org/10.1016/ S14702045(20)30388-0.

15. Chile. Source: Globocan 2018. International Agency for Research on Cancer. WHO.

16. Vanni G, Pellicciaro M, Materazzo M, Palombi L, Buonomo O. Breast Cancer Diagnosis in Coronavirus-Era: Alert From Italy. Frontiers in Oncology. 2020;10:1-2. doi: $10.3389 /$ fonc. 2020.00938 . 